\title{
Evaluación de un programa de intervención basado en el uso de las TIC para mejorar la satisfacción del alumnado hacia la Educación Física
}

\author{
Evaluation of an intervention program based on the use of ICT to \\ increase the satisfaction of students regard to Physical Education
}

\author{
Manuel Luis Sánchez Sánchez \\ María Espada Mateos \\ Universidad Politécnica de Madrid
}

Recibido: 23/10/2017

Aceptado: 20/06/2018

\begin{abstract}
Education is evolving and ICT (Information and Communication Technology) is an important part of this evolution. However, in Physical Education there is a barrier to the use of this technology since it is mainly associated with conceptual contents and Physical Education is principally a procedural subject. The main goal of this research was to evaluate the satisfaction of Physical Education students in the use of ICT. An intervention was thus carried out with a sample of 107 students from two schools, aged between 14 and 17 years $(X=14.95, \mathrm{DT}=0.49)$ and 15 and 18 years of age $(X=16.10, S D=0.73)$, divided into an experimental group of 63 students and a control group of 44 students. A questionnaire was used to evaluate their level of satisfaction with the Physical Education lessons (PACSQ). The results confirm that there is a close relationship between the use of ICT in Physical Education lessons and an increase in students' satisfaction.
\end{abstract}

KEY WORDS: motivation, ICT, Physical Education, teenagers, intervention

\section{RESUMEN}

La educación sigue una evolución en la que las TIC (Tecnologías de la Información y Comunicación) son una parte fundamental de la misma. Sin embargo, en la asignatura de Educación Física hay una barrera para la utilización de las TIC ya que su uso se asocia principalmente a contenidos conceptuales, siendo la Educación Física una materia de carácter principalmente procedimental. El objetivo de este estudio es evaluar la satisfacción de los estudiantes hacia el uso de las TIC en la asignatura de Educación Física. Para ello se ha realizado una intervención con una muestra de 107 estudiantes de dos centros educativos, divididos en un grupo experimental de 63 alumnos y un grupo control de 44 alumnos, con edades comprendidas entre 14 y 17 años $(X=14,95$; DT $=0,49)$ y 15 y 18 años de edad $(X=16,10$; $\mathrm{DT}=0,73)$. Se utilizó un cuestionario para evaluar el nivel de satisfacción hacia las clases de Educación Física (PACSQ). Los resultados confirman que existe una estrecha relación entre la utilización de las TIC en las clases de Educación Física y el aumento de la satisfacción en los estudiantes.

PALABRAS CLAVE: motivación, Educación Física, adolescentes, intervención

Authors' correspondence address:

María Espada Mateos Universidad Politécnica de Madrid. Calle Martín Fierro, 7, 28040Madrid

E-mail: maria.espada@upm.es. ORCID: orcid.org/0000-0002-3815-1037 


\section{Introducción}

La implicación en actividades físicas tanto en el ámbito escolar como en su tiempo libre en los adolescentes ha disminuido durante las últimas décadas (Pate et al., 2006).

Por este motivo, se han buscado estrategias eficaces que ayuden a que la actividad física forme parte de la vida de los adolescentes. Un aspecto que puede aportar mucha diferencia en un estilo de vida activo, es motivarles de forma óptima en la asignatura de Educación Física (Haerens, Kirk, Cardon, De Bourdeaudhuij \& Vansteenkiste, 2010). Entendiendo esta motivación, como el interés en el aprendizaje (Ibáñez \& Fernández, 2013).

En esta línea, Pérez Pueyo (2016) introduce el denominado Estilo Actitudinal, con el cual pretende conseguir una mayor motivación hacia la Educación Física y, consecuentemente, un mejor aprendizaje de la misma.

Por su parte, Moreno, Cervelló \& Martínez (2007), introdujeron la motivación en la Teoría de la Autodeterminación (TAD), la cual representa los aspectos de desarrollo de cada acción, según el orden en el cual se van desarrollando en el tiempo. La TAD está aplicada dentro de la motivación para medir el grado de interés hacia la actividad física. El mayor grado de determinación lo alcanzan aquellos deportistas que se encuentran intrínsecamente motivados, lo que conlleva a practicar actividad física por placer y disfrute (Deci \& Ryan, 2000; Granero-Gallegos, Gómez-López, BaenaExtremera, Abraldes \& Rodríguez-Suárez, 2012).

En las clases de Educación Física existen diferencias en cuanto a la satisfacción del alumnado según el modo en que el profesorado aborda los contenidos (Mouratidis, Vansteenkiste, Lente \& Sideridis, 2011).De esta forma, algunos estudiantes realizan las actividades porque les gusta, por diversión, mientras que otros alumnos muestran un nivel de inactividad bastante elevado que hace de estas clases una simple distracción en relación a otras asignaturas (Cox, Smith \& Williams, 2008).

Por otra parte, hay que destacar las diferencias actuales entre géneros dentro de esta modalidad. Tal y como indican Lyu \& Gill (2011), la satisfacción hacia la práctica deportiva puede variar en función del género. En esta línea, un reciente estudio elaborado por Espada \& Galán (2017) concluye que las chicas tienen peor autoconcepto y motivación hacia la práctica de actividades físico-deportivas que los chicos.

Por otra parte, la rápida integración de tecnologías de la información y comunicación (TIC) en distintos contextos sociales, ha ampliado las oportunidades de aprender más allá de las fronteras definidas durante muchos años por los sistemas educativos tradicionales (Cerdá, López, Osses y Saiz, 2015). La incorporación de las TIC en el ámbito educativo, están aportando cambios significativos en los estudiantes. De esta forma, se afirma que los alumnos que utilizan estos recursos muestran un mayor conocimiento sobre el contenido a estudiar, que los que siguen el método tradicional. Por lo tanto, el papel del profesor que da órdenes y dirige a los alumnos pasa a un segundo plano, convirtiéndole en un apoyo para entender los contenidos a través de estas nuevas tecnologías (Volman, 2005). En esta línea, Jiménez, Mora \& Cuadros (2016) exponen que los profesores deberían cambiar la metodología tradicional para adaptarse al ritmo y las necesidades de los alumnos digitales. Estos autores señalan que es un hecho que las formas tradicionales de interacción han cambiado con las TIC; de la interacción cara a cara entre los interlocutores se ha pasado a una interacción entre los alumnos, que puede ser sincrónica (en tiempo real: chat, vídeo) o asincrónica (en tiempo no real: correo electrónico, foro).

El uso actual de las tecnologías hace referencia al aprendizaje, tanto dentro como fuera del aula didáctica, para que los estudiantes utilicen las TIC como forma de vida y para fines tanto profesionales como vocacionales (Drent \& Meelissen, 2008). El uso de las TIC en la educación permite la creación de ambientes educativos, motivantes y retadores para la adquisición de conocimientos (Delgado, Arrieta \& Riveros, 2009).

El docente, con el uso de tecnologías, aumenta sus posibilidades y capacidad para actuar en el marco del aula, obteniendo recursos útiles para su desempeño profesional, además de resultar un instrumento motivador para el alumnado de la educación obligatoria (Ruíz López, 2015). Los profesores de Educación Física tienen la posibilidad de utilizar las TIC para facilitar el aprendizaje, pueden utilizarse para mejorar la calidad de la asignatura a través de elementos con cámaras digitales, 
videos, Apps para transmitir información como bailes aeróbicos, resultados fisiológicos y ejemplos de acciones motrices deportivas para desarrollar los contenidos de enseñanza (Collins, 2011).

La actitud del profesorado a la hora de utilizar estas TIC es importante, pero se pueden encontrar en ocasiones con que es el alumnado el que carece de motivación hacia la asignatura (Álvarez, González \& García, 2008).

Sin embargo, aunque las ventajas de estas metodologías son mostradas por los profesores a los alumnos, su uso a nivel práctico sigue siendo muy eventual y preciso según el tipo de actividad que se realice, es decir, su desarrollo presenta diversas limitaciones implícitas en los procesos de aprendizaje colaborativo cognitivo. Entre ellas se apunta, por ejemplo, la complejidad que supone para los docentes la planificación de actividades de trabajo colaborativo a través de las herramientas tecnológicas, porque es preciso diseñar y organizar cuidadosamente la actividad teniendo en cuenta el breve tiempo del que se dispone, y prever posibles problemas de comunicación que pueden generarse entre los estudiantes durante su desarrollo (Hernández Martín \& Martín de Arriba, 2017).

Otras limitaciones a las que apuntan distintos estudios se relacionan directamente con la necesidad de un tiempo adicional que en ocasiones no se tiene para gestionar la comunicación con los alumnos en torno a las TIC. En este sentido, como señalan García-Valcárcel, Basilotta \& López (2014) lejos de ahorrar tiempo, el uso de las TIC puede llegar a restarlo para dedicarse a otro tipo de tareas que oficialmente se le reconocen al docente.

Barahona (2012) afirma que las TIC son aconsejables para el progreso de la educación, ya que permiten desarrollar una competencia digital para el aprendizaje autónomo entre estudiantes, enriquece el aprendizaje a través de búsquedas de información en la red o para diversificar las tareas en el contexto enseñanza-aprendizaje. Además, hacen posible el acceso rápido y eficaz a la información de manera más atractiva e innovadora para después compartirla. Una de las mayores ventajas de usar estas tecnologías es que rompe las barreras espacio-temporales que tanto condicionan la enseñanza en Educación Física.

Tal y como expone Tahara \& Darido (2016) la literatura científica que se centra en la relación del uso de las TIC en las clases de educación física, aunque está aumentando en los últimos años, es todavía escasa. Por ello, el objetivo principal de la presente investigación es evaluar si la utilización de las TIC aumenta la satisfacción hacia las clases de Educación Física. Además, se ha analizado las diferencias en función del género.

\section{Metodología}

Este estudio presenta un diseño cuasi-experimental, de enfoque cuantitativo a través del método de la encuesta (Cea, 2010; Thyer, 2012).

\subsection{Participantes}

Para llevar a cabo este estudio se tomó una muestra incidental total de 107 alumnos entre los dos colegios. En ambos centros, se tomaron dos grupos en las clases de Educación Física: el grupo experimental con un total de 63 alumnos, con edades comprendidas entre 14 y 17 años $(X=14,95$; $\mathrm{DT}=0,49)$ y el grupo control con 44 alumnos, de edades comprendidas entre 15 y 18 años de edad $(\mathrm{X}=16,10 ; \mathrm{DT}=0,73)$.

\subsection{Instrumentos / Materiales}

Runtastic es una aplicación para hacer deporte. Cuenta con funciones básicas como seguimiento por vía GPS, mapa de la zona, historial de recorridos, entrenamientos y estadísticas. Runtastic es también una red social de aptitud, ya que comparte los progresos que los usuarios hacen por las redes sociales a través de Facebook, Google y Twitter. Una vez que se haya seleccionado el tipo de entrenamiento, se puede elegir el tipo de actividad, desde correr, ciclismo, patinaje, raquetas y tenis de mesa. Cuando se escoge el tipo de actividad, se presiona el botón "iniciar ejercicio". La aplicación pide activar siempre el GPS para medir bien el rimo y la distancia. También se puede conectar con personas online que están entrenando en ese momento, y se les puede añadir 
comentarios alentadores para motivarlos. Durante el entrenamiento aparecen cuatro indicadores principales que permiten seguir el ritmo, la velocidad, la distancia y las calorías. También se pueden intercambiar módulos y de esta forma activar otros indicadores como velocidad media, ritmo promedio, ganancia (en el caso de la escalada) pérdida, elevación, ritmo cardíaco, etc. (Castro, 2013).

Heart Rate es una aplicación que mide el pulso cardiaco a través de la cámara del teléfono móvil. Para una mayor precisión, se necesita tener flash en la cámara (que se encenderá para la prueba), aunque también se puede medir en una habitación muy bien iluminada. Para que el proceso sea fiable, se debe poner el dedo sobre el flash de la cámara y esperar 10 segundos. De esta forma, el móvil capta los cambios de la luz en el dedo y realiza el cálculo. Concretamente, mide los cambios de saturación en el oxígeno de la sangre. Las fluctuaciones que se producen en la saturación con cada latido del corazón, son las que provocan un ligero cambio en el color de la piel. Runtastic Heart Rate también cuenta con un servicio de historial para ver la hora y el día de la medición, así como si fue realizada en reposo o después de haber realizado ejercicio físico. También podremos incluir las sensaciones después de la medición mediante un sencillo selector visual con 5 iconos. Runtastic Heart Rate cuenta con la posibilidad de compartir nuestros resultados en Facebook, Twitter y Mail. Evidentemente, la calidad de la medición no es del todo correcta si se compara con un pulsímetro tradicional (Merino, 2013).

Para medir la motivación y el grado de interés de los alumnos hacia estas Apps y su relación con la unidad didáctica en la que se han utilizado, se ha utilizado el cuestionario PACQS (Sicilia, Ferriz, Trigueros \& González Cutre, 2014).

Este instrumento examina la satisfacción en las clases de Educación Física. Este cuestionario fue una versión adaptada al español de Physical Activity Class Satisfaction Questionnaire (Cunningham, 2007). El cuestionario está encabezado por la frase: "Indica tu nivel de satisfacción con las clases de Educación Física recibidas respecto a....", y consta de 33 ítems repartidos en nueve factores. Se responde y se evalúa por medio de la escala Likert desde el 1 (totalmente desacuerdo) al 8 (totalmente de acuerdo).

Para analizar su fiabilidad y validez se hizo un análisis de sus propiedades psicométricas en las cuales, el peso de regresión estándar fue de $\mathrm{p}<0.001$, siendo de 0.72 para enseñanza, 0.86 para la relajación, 0.91 para desarrollo cognitivo, 0.95 para mejora de la salud, 0.78 para interacción con otros, 0.59 de éxito normativo, 0.81 para diversión y disfrute, 0.97 para experiencias de maestría y 0.99 experiencias recreativas (Sicilia, Ferriz, Trigueros \& González Cutre, 2014).

Los autores realizaron un análisis de consistencia interna, que reveló valor alfa de Cronbach de 0.89 para la subescala de enseñanza; 0.9 para relajación; 0.91 para desarrollo cognitivo; 0.92 para mejora de la salud; 0.8 para interacción con otros; 0.89 para éxito normativo; 0.88 para diversión y disfrute; 0.86 para experiencias de maestría y 0.89 para experiencias recreativas. También se basó en un análisis de estabilidad temporal, siendo de 0.84 para enseñanza, 0.76 para la relajación, 0.82 para desarrollo cognitivo, 0.83 para mejora de la salud, 0.73 para interacción con otros, 0.79 de éxito normativo, 0.81 para diversión y disfrute, 0.78 para experiencias de maestría y 0.77 en experiencias recreativas (Sicilia, Ferriz, Trigueros \& González Cutre, 2014).

\subsection{Método}

Se desarrollaron dos tipos de grupos: uno experimental, que abarca $4^{\circ} \mathrm{ESO}$ con 31 estudiantes (17 chicos y 14 chicas) y $1^{\circ}$ Bachillerato con 32 alumnos (18 chicos y 14 chicas), en el cual se utilizaron las TIC durante el desarrollo de las clases de Educación Física; y un grupo control, que estaba compuesto por aquellos alumnos que iban a continuar con sus clases de forma regular y sin intervención alguna, formado por $4^{\circ} \mathrm{ESO}$ con 11 alumnos $\left(5\right.$ chicos y 6 chicas) y por $1^{\circ}$ Bachillerato con 33 alumnos (19 chicos y 14 chicas).

La unidad didáctica llevada a cabo en este estudio fue la misma en ambos colegios: la condición física, concretamente se trató el tema de la resistencia aeróbica y anaeróbica, los métodos de carrera y la flexibilidad. Esta unidad se desarrolló desde contenidos que van desde las distintas formas de 
resistencia, pasando por el método "Farlek", entrenamientos como "el Julio" y "los 10 mandamientos" hasta finalmente llegar al "entrenamiento total".

El estudio fue realizado de un modo analítico para que se apreciaran los contenidos de la unidad didáctica a través de las TIC en dicha unidad, de la condición física. Se llevó a cabo durante los meses Octubre y Noviembre de 2016, con un máximo de 6 clases, con 2 sesiones por semana en cada instituto. Los contenidos impartidos en ambos grupos fueron los mismos. Sin embargo, en el grupo experimental aprendieron contenidos conceptuales y procedimentales a través del uso de las aplicaciones tecnológicas.

Previamente al desarrollo de las sesiones, se repartió una autorización a todos los alumnos para permitir el uso de las TIC durante las clases de Educación Física, y su autorización para participar en el estudio. También se repartió el anterior y mencionado cuestionario de satisfacción hacia las clases de Educación Física. Este instrumento se pasó antes y después de las sesiones para medir la diferencia de satisfacción antes y después de finalizar la unidad didáctica. Finalmente, antes de comenzar con la primera sesión, se les informó a los alumnos de los grupos experimentales, de ambos colegios, sobre las características de las Apps Runtastic y Hear Rate, se les pidió que se las descargaran y se registraran en ellas para empezar a utilizarlas de forma inmediata.

Cada profesor impartiría sus clases en el grupo experimental con la condición de utilizar esta tecnología en sus ejercicios. Los contenidos de estas sesiones fueron los mismas para ambos colegios. De este modo se podría comparar con mayor exactitud las diferencias motivaciones en ambos grupos.

\subsection{Análisis de datos}

Tras realizar la prueba de normalidad de la muestra a través del test de Kolmogorov Smirnov (K-S de muestra), las variables no presentan una distribución normal; por lo que se utilizan estadísticos no paramétricos para el análisis de las variables. Para comprobar las diferencias intragrupo en la toma pre-test y en la toma post-test, se llevó a cabo la prueba de Wilcoxon para muestras relacionadas con cada una de los grupos. Posteriormente, para analizar las diferencias por sexo, se llevó a cabo el test U Mann-Whitney para muestras independientes.

\section{Resultados y discusión}

Para analizar los efectos de la intervención en cada grupo se llevó a cabo una prueba para muestras relacionadas, prueba de Wilcoxon, de los diferentes factores, con la toma pre-test y posttest, para así las diferencias entre ellas. El análisis de los datos reflejó en el grupo experimental, una mayor diferencia de motivación tras la intervención en enseñanza $(p=.00)$, relajación $(p=.00)$, desarrollo cognitivo $(\mathrm{p}=.00)$, mejora de salud $(\mathrm{p}=.00)$, interacción con otros $(\mathrm{p}=.00)$, diversión y disfrute $(\mathrm{p}=.00)$, experiencia del maestro $(\mathrm{p}=.00)$ y experiencias recreativas $(\mathrm{p}=.00)$. Sin embargo, la puntuación en el grupo control en éxito normativo $(p=.00)$ fue superior que la del grupo experimental en el pre-test y el post-test. También cabe apreciar, que las mayores puntuaciones entre ambas tomas son registradas en el grupo experimental con las variables enseñanza, desarrollo cognitivo, mejora de salud, diversión y disfrute y experiencias recreativas con más de 5 puntos de media, siendo la variable desarrollo cognitivo la más destacada de todas.

Tabla 1

Wilcoxon de la toma pre-test y la toma post-test entre el grupo experimental y el grupo control.

\begin{tabular}{|c|c|c|c|c|c|c|c|c|c|c|c|}
\hline \multirow{2}{*}{$\begin{array}{l}\text { GRUPOS } \\
\text { VARIABLES }\end{array}$} & & \multicolumn{2}{|c|}{$\begin{array}{l}\text { EXPERIMENTAL } \\
(\mathrm{N}=63)\end{array}$} & \multicolumn{2}{|c|}{$\begin{array}{c}\text { CONTROL } \\
(\mathrm{N}=44)\end{array}$} & \multicolumn{2}{|c|}{ WILCOXON } & \multicolumn{2}{|c|}{ SIG } & \multicolumn{2}{|c|}{ TOTAL } \\
\hline & TIPO & $\mathrm{X}$ & DT & $\bar{X}$ & DT & EXP & CNT & EXP & CNT & WILCOX & SIG \\
\hline ENSEÑANZA & PRE-TEST & 21,49 & 4,846 & 21,58 & 3,874 & & & & & & \\
\hline & POST-TEST & 27,16 & 2,371 & 24,40 & 2,921 & $-5,954$ & - &, 000 &, 000 & $-7,416$ &, 000 \\
\hline RELAJACIÓN & PRE-TEST & 15,60 & 3,439 & 16,56 & 2,119 & & & & & & \\
\hline & POST-TEST & 19,84 & 2,548 & 18,88 & 2,174 & $-6,158$ & $\overline{4}, 394$ &, 000 &, 000 & $-7,673$ &, 000 \\
\hline DESARROLLO & PRE-TEST & 25,62 & 4,397 & 26,30 & 4,234 & & & & & & \\
\hline COGNITIVO & POST-TEST & 33,62 & 3,400 & 30,60 & 3,353 & $-6,469$ & - &, 000 &, 000 & $-8,001$ &, 000 \\
\hline
\end{tabular}




\begin{tabular}{|c|c|c|c|c|c|c|c|c|c|c|c|}
\hline MEJORA SALUD & $\begin{array}{l}\text { PRE-TEST } \\
\text { POST-TEST }\end{array}$ & $\begin{array}{l}25,37 \\
32,84\end{array}$ & $\begin{array}{l}4,598 \\
3,516\end{array}$ & $\begin{array}{l}26,05 \\
30,93\end{array}$ & $\begin{array}{l}4,254 \\
3,112\end{array}$ & $-6,463$ & - & ,000 &, 000 & $-8,232$ & ,000 \\
\hline INTERACCIÓN & $\begin{array}{l}\text { PRE-TEST } \\
\text { POST-TEST }\end{array}$ & $\begin{array}{l}16,86 \\
20,48\end{array}$ & $\begin{array}{l}2,988 \\
1,804\end{array}$ & $\begin{array}{l}17,44 \\
19,72\end{array}$ & $\begin{array}{l}2,889 \\
2,119\end{array}$ & $-5,745$ & $\overline{4,284}$ & ,000 &, 000 & $-7,346$ & ,000 \\
\hline ÉXITO NORMA & $\begin{array}{l}\text { PRE-TEST } \\
\text { POST-TEST }\end{array}$ & $\begin{array}{l}12,14 \\
16,17\end{array}$ & $\begin{array}{l}4,697 \\
5,777\end{array}$ & $\begin{array}{l}14,00 \\
16,70\end{array}$ & $\begin{array}{l}3,505 \\
4,323\end{array}$ & $-5,783$ & - & ,000 &, 000 & $-7,233$ & ,000 \\
\hline DIVERSIÓN & $\begin{array}{l}\text { PRE-TEST } \\
\text { POST-TEST }\end{array}$ & $\begin{array}{l}15,76 \\
20,25\end{array}$ & $\begin{array}{l}3,800 \\
2,416\end{array}$ & $\begin{array}{l}16,81 \\
19,00\end{array}$ & $\begin{array}{l}2,962 \\
2,320\end{array}$ & $-5,804$ & 3,518 & ,000 &, 000 & $-6,849$ & ,000 \\
\hline EXP. MAESTRO & $\begin{array}{l}\text { PRE-TEST } \\
\text { POST-TEST }\end{array}$ & $\begin{array}{l}15,17 \\
19,84\end{array}$ & $\begin{array}{l}3,382 \\
2,424\end{array}$ & $\begin{array}{l}16,51 \\
18,21\end{array}$ & $\begin{array}{l}2,530 \\
2,642\end{array}$ & $-6,160$ & - & ,000 &, 000 & $-7,274$ & ,000 \\
\hline EXP.RECREATIVA & $\begin{array}{l}\text { PRE-TEST } \\
\text { POST-TEST }\end{array}$ & $\begin{array}{l}20,44 \\
26,76\end{array}$ & $\begin{array}{l}4,099 \\
3,009\end{array}$ & $\begin{array}{l}21,60 \\
24,67\end{array}$ & $\begin{array}{l}3,325 \\
3,145\end{array}$ & $-6,316$ & $\begin{array}{l}- \\
4,148\end{array}$ & ,000 &, 000 & $-7,723$ & ,000 \\
\hline
\end{tabular}

En segundo lugar, se realizó una prueba U Mann-Whitney para 2 muestras independientes para analizar así las diferencias entre hombres y mujeres. El análisis de los datos reflejó que en el grupo de los hombres existe mayor puntuación de motivación en enseñanza, relajación, desarrollo cognitivo, mejora de salud, interacción con otros, éxito normativo, diversión y disfrute, experiencia del maestro y experiencias recreativas. No obstante, la relación es estadísticamente significativa únicamente en éxito normativo $(\mathrm{p}=.000)$ y experiencias recreativas $(\mathrm{p}=.003)$.

Tabla 2

U Mann-Whitney de la toma pre-test y la toma post-test entre hombres y mujeres

\begin{tabular}{|c|c|c|c|c|c|}
\hline \multirow{2}{*}{ HOMBRE $(\mathrm{N}=59)$} & \multirow{2}{*}{ MUIER ( $\mathrm{N}=46)$} & \multicolumn{2}{|c|}{ DESCRIPTIVOS } & \multicolumn{2}{|c|}{ U MANN-WHITNEY } \\
\hline & & & & & \\
\hline VARIABLES & SEXO & $\mathrm{X}$ & $\overline{\text { DT }}$ & $\mathrm{U}$ & SIG \\
\hline \multirow[t]{2}{*}{ ENSEÑANZA } & HOMBRE & 26,54 & 3,126 & & \\
\hline & MUJER & 25,37 & 2,576 & 999,500 & ,020 \\
\hline \multirow[t]{2}{*}{ RELAJACIÓN } & HOMBRE & 19,90 & 2,115 & & \\
\hline & MUJER & 18,85 & 2,724 & 1015,000 & 025 \\
\hline \multirow{2}{*}{$\begin{array}{l}\text { DESARROLLO } \\
\text { COGNITIVO }\end{array}$} & HOMBRE & 32,07 & 3,807 & & \\
\hline & MUJER & 32,69 & 3,543 & 1178,500 & ,246 \\
\hline \multirow[t]{2}{*}{ MEJORA SALUD } & HOMBRE & 32,56 & 3,500 & & \\
\hline & MUJER & 31,48 & 3,404 & 1111,000 &, 110 \\
\hline \multirow[t]{2}{*}{ INTERACCIÓN } & HOMBRE & 20,41 & 1,877 & & \\
\hline & MUJER & 19,83 & 2,047 & 1096,000 & 087 \\
\hline \multirow[t]{2}{*}{ ÉXITO NORMA } & HOMBRE & 19,14 & 2,467 & & \\
\hline & MUJER & 13,02 & 5,748 & 562,000 &, 000 \\
\hline \multirow[t]{2}{*}{ DIVERSIÓN } & HOMBRE & 20,07 & 2,592 & & \\
\hline & MUJER & 19,37 & 2,225 & 1081,500 &, 072 \\
\hline \multirow[t]{2}{*}{ EXP. MAESTRO } & HOMBRE & 19,58 & 2,465 & & \\
\hline & MUJER & 18,63 & 2,776 & 1103,000 & 097 \\
\hline \multirow[t]{2}{*}{ EXP.RECREATIVA } & HOMBRE & 26,63 & 2,888 & & \\
\hline & MUJER & 24,96 & 3,425 & 907,500 &, 003 \\
\hline
\end{tabular}

\section{Discusión}

El objetivo que se ha planteado en este estudio ha sido comprobar si existe un aumento en la satisfacción hacia las clases de Educación Física tras realizar una intervención con TIC, con el fin de 
analizar la necesidad de innovación de estas tecnologías en el entorno académico y concretamente en dicha asignatura.

A nivel general se puede observar, que los resultados en el grupo experimental son superiores que en el grupo control en todas las dimensiones después de haber realizado la intervención, demostrando que los alumnos ponen más interés en la asignatura de la Educación Física cuando utilizan las TIC.

Los resultados estadísticos de ambas tomas, muestran que la variable interacción entre los alumnos en clase para trabajar en equipo, presenta mejor puntuación en el grupo experimental que en el control, tras realizar la intervención. Si relacionamos estos resultados con los del estudio de Vansteenkiste et al. (2012), demuestran que los estudiantes de Educación Secundaria se sienten más motivados en las clases si trabajan en equipo manteniendo la atención en las clases, tal y como sucedía en la presente investigación, en la cual el alumnado ha tenido que trabajar en equipo otros a través de las Apps para móviles.

Además, los alumnos del grupo experimental aceptan en mayor grado que aprenden mejor la asignatura y afirman que les gusta trabajar en grupo para realizar actividades, aprenden mejor con la ayuda de sus compañeros y las herramientas digitales son imprescindibles para un análisis más profundo de la materia, mostrando así más interés por los contenidos y por aprender cosas nuevas. Además, se desaniman menos cuando tienen muchos temas que aprender e intentan prestar ayuda cuando ven que un compañero tiene dificultades con algún tema.

Por lo tanto, se puede afirmar que la enseñanza usando las TIC, tiende a aumentar la satisfacción de los alumnos en las clases de Educación Física. Corroborando lo expuesto por Aguaded y Tirado (2010), quienes determinan que la utilización de las TIC permite que las clases se vuelven más activas y participativas, provoca un aumento de la motivación de los alumnos que se traduce en una actitud más propicia hacia las tareas académicas propuestas por el profesor, mejorando la atención a sus indicaciones y su implicación en el aprendizaje. Consecuentemente, los alumnos no solo buscan incrementar su saber y sus capacidades y experimentar su competencia, sino también poder ayudar a otros.

Además, es importante resaltar que el grupo experimental presenta mayores puntuaciones en cuanto a la experiencia recreativa y la diversión, esto podría deberse a que el uso de las TIC en esta intervención ha promovido la cooperación y la colaboración entre ellos. En esta línea, Huertas y Pantoja (2016) señalan que la formación basada en las TIC permite a los estudiantes que vayan a su propio ritmo de aprendizaje, pero fomenta el estilo grupal sobre el individual. Además, desde un punto de vista pedagógico, se afirma que el conocimiento es un proceso activo de construcción personal de la información y tiende a ser interactiva tanto entre los participantes en el proceso de aprendizaje como con los contenidos de la unidad didáctica.

La valoración positiva de las TIC en el desarrollo de estrategias de aprendizaje eficaces se encuentra también en consonancia con el estudio de García-Valcárcel \& Tejedor (2017) quienes afirman que las tecnologías promueven el aprendizaje autónomo, el trabajo colaborativo, el pensamiento creativo y el trabajo interactivo.

Respecto a las diferencias en función del género, se pudo observar que los chicos presentan mayores puntuaciones que las chicas en todos los factores. Cabe destacar que en el desarrollo cognitivo tanto los chicos como las chicas tienen casi la misma puntuación media y en éxito normativo es el factor en el que más diferencia de medias existe. Estos resultados podrían deberse, a que tal y como exponen Muñoz, Campos, Toro \& de Los Fayos (2012), puedan existir actitudes sexistas en el ámbito del deporte con respecto a las niñas, lo que influye en una menor motivación hacia la asignatura de Educación Física. Coincidiendo con los datos del estudio elaborado por Espada y Galán (2017) en el cual se determina que las chicas tienen menos motivación que los chicos para la práctica deportiva.

Si analizamos de forma más exhaustiva estos datos, las chicas presentan puntuaciones medias inferiores frente al chicos en la mayoría de las dimensiones, lo cual determina que tienen menos satisfacción hacia las clases de Educación Física. No obstante, a pesar de que los resultados han sido desfavorables para las chicas, se ha demostrado que las mujeres presentan más automotivación en el 
post-test, lo cual indica que las chicas han aumentado su satisfacción hacia las clases de Educación Física gracias el uso de las TIC.

Otro aspecto que podría explicar estas diferencias en la satisfacción en las clases de Educación Física en función del género es los contenidos tratados en la intervención ya que se trabajaron aspectos relacionados con las capacidades físicas. Teniendo en cuenta los resultados del estudio de Sevil, Abós, Julián, Murillo \& García-González (2015) las chicas presentan mejores pronósticos de puntuación en actividades más cercanas al acrosport o actividades físico artístico expresivas.

Estos resultados nos llevan a la reflexión de que, en la actualidad, continúa observándose algunos síntomas de una educación más sexista en los niños que en las niñas, donde los estereotipos de género siguen estando muy presentes, aspecto que también se recoge en el trabajo de Díaz-Aguado y Martín (2011). Estas conductas se podrían explicar por la presencia e influencia de modelos parentales que las transmiten de generación en generación, en el marco de una sociedad que contribuye a la formación de estos estereotipos (Vázquez, 2010). Además, estas creencias y actitudes pueden estar repercutiendo en una menor participación de la mujer en el deporte, limitando así sus posibilidades para desarrollar su habilidad física (Blackwood, 2010), y desfavorecer la categorización de deportes considerados como masculinos y femeninos.

Así, en el estudio de Borges, Belando \& Moreno-Murcia (2013), se analizó la relación entre las creencias implícitas que predicen la capacidad atlética, el nivel de autodeterminación, la discriminación percibida, la percepción de igualdad de trato y su importancia y utilidad otorgada a la Educación Física. En dicho estudio se concluyó que los profesores deberían promover la creencia de que se pueden mejorar las habilidades deportivas a través de la participación en de aprendizaje, el esfuerzo y el trabajo de grupo en las clases de educación física, ya que podría alentar a los estudiantes a tener una mayor percepción de igualdad de trato.

\section{Conclusiones}

Como conclusiones se puede extraer que los alumnos del grupo experimental presentan de forma significativa mayores rasgos de satisfacción hacia las clases de Educación Física respecto al grupo control. Esto se debe a que el grupo experimental utilizo las TIC durante las clases de Educación Física.

En relación al género, los chicos presentan una puntuación media superior que las chicas, lo que permite afirmar que los chicos presentan de forma significativa mayores rasgos de satisfacción hacia las clases de Educación Física.

Además, se anima a realizar futuras investigaciones siguiendo la línea de este trabajo, aplicando las TIC en otras unidades didácticas que desarrollen contenidos diferentes de la asignatura de Educación Física para una mayor precisión en los niveles de satisfacción de los alumnos así como en las diferencias de motivación en función del sexo ya que en investigaciones previas se ha constatado que las chicas presentan mayores índices de motivación hacia los contenidos relacionados con las actividades físico artístico expresivas.

\section{Referencias bibliográficas}

Aguaded, J. I. y Tirado, M. (2010). Ordenadores en los pupitres: informática y telemática en el proceso de enseñanza-aprendizaje en los centros TIC de Andalucía. Píxel-Bit. Revista de medios y educación, 36, 5-28.

Alonso Tapia, J. (2005). Motivaciones, expectativas y valores - intereses relacionados con el aprendizaje: el cuestionario MEVA. Psicothema, 17, 404-411.

Álvarez, B., González, C. \& García, N. (2008). La motivación y los métodos de evaluación como variables fundamentales para estimular el aprendizaje autónomo. Revista de Docencia Universitaria. 2, $1-12$. 
Barahona, J.D. (2012). The Teaching of Physical Education implemented with ICT. Educación Física y Deporte. 31 (2), 1047-1056.

Borges, F., \& Belando, N., \& Moreno-Murcia, J. (2014). Percepción de igualdad de trato e importancia de la educación física de alumnas adolescentes. Revista de Psicodidáctica, 19 (1), 173-189.

Blackwood, T. (2010). Playing baseball/playing 'house': The reproduction and naturalization of 'separate spheres' in Japanese high school baseball. Sport, Education and Society, 15(1), 83-101.

Castro, M. (2013). Runtastic Pro: análisis de la aplicación para deportistas. Recuperado de www.computerhoy.com.

Cea, M.A. (2010). Métodos de encuesta. Teoría y práctica, errores y mejora. Madrid: Síntesis.

Cerdá, C. López, O. Osses, S. y Saiz, J.L. (2015). Análisis Psicométrico de la Escala de Aprendizaje de Autodirigido Basada en la Teoría de Aprendizaje Autodirigido de Garrison. Revista Iberoamericana de Diagnóstico y Evaluación - e Avaliação Psicológica. RIDEP. 39 (1), 46-56

Collins, O. (2011). The use of ICT in teaching and learning of physical education. Continental Journal of Education Research. 4(2), 29 -32.

Cox, A.E., Smith. A.L. \& Williams, L. (2008). Change in physical education motivation and physical activity behavior during middle school. The Journal of Adolescent Health. 43(5), 506-513

Cunningham, G. B. (2007). Development of the physical activity class satisfaction questionnaire (PACSQ). Measurement in Physical Education and Exercise Science. 11(3), 161-176.

Deci, E.L. \& Ryan, R.M. (2000). The "what" and "why" of goal pursuits: Human needs and the selfdetermination of behaviour. Psychological Inquiry, 11, 227- 268.

Delgado, M. Arrieta, X. \& Riveros, V. (2009). Uso de las TIC en educación, una propuesta para su optimización. Omnia, 15(3), 58-77.

Díaz-Aguado, M. J. y Martín, G. (2011). Convivencia y aprendizaje escolar en la adolescencia desde una perspectiva de género. Psicothema, 23(2), 252-259

Drent, M. \& Meelissen, M. (2008). Which factors obstruct or stimulate teacher educators to use ICT innovatively? Computers \& Education. 51 (1), 187-199.

Espada, M. Y Galán, S. (2017). Evaluación de las barreras para la práctica de actividad física y deportiva en los adolescentes españoles. Revista Salud Pública, 19(6), 743-747.

García-Valcárcel, A. Basilotta, V. \& López, C. (2014). Las TIC en el aprendizaje colaborativo en el aula de Primaria y Secundaria, Comunicar, 42, 65-74.

García-Valcárcel, A. y Tejedor Tejedor, F.J. (2017). Percepción de los estudiantes sobre el valor de las TIC en sus estrategias de aprendizaje y su relación con el rendimiento. Educación XX1 20(2), 137-159, doi:10.5944/educXX1.13447

Granero-Gallegos, A., Gómez-López, M., Baena-Extremera, A., Abraldes, J.A. \& Rodríguez-Suárez, N. (2012). La motivación autodeterminada en el balonmano amateur. Revista Iberoamericana de Diagnóstico y Evaluación - e Avaliação Psicológica. RIDEP. 33 (1), 147-171

Haerens, L., Kirk, D., Cardon, G., De Bourdeaudhuij, I. \& Vansteenkiste. M. (2010). Motivational profiles for secondary school physical education and its relationship to the adoption of a physically active lifestyle among university students. European Physical Education Review. 16 (2), 117-139.

Hernández Martín, A. \& Martín de Arriba, J. (2017). Concepciones de los docentes no universitarios sobre el aprendizaje colaborativo con TIC. Educación XX1, 20(1), 185-208,

Huertas, A. \& Pantoja, A. (2016). Efectos de un programa educativo basado en el uso de las TIC sobre el rendimiento académico y la motivación del alumnado en la asignatura de tecnología de educación secundaria. Educación XX1. 19 (2), 229-250.

Ibáñez, D. \& Fernández, M. (2013). La motivación en la enseñanza de la danza: análisis de la teoría de la autodeterminación. Joumal for educators, teachers and trainers. 5 (1), 124-138.

Jiménez, D., Mora, M. \& Cuadros, R. (2016). La importancia de las nuevas tecnologías en el proceso educativo. Propuesta didáctica TIC para ELE: mELEndien7dias. Revista Fuentes, 18(2), 209-223. Doi: http:/ /dx.doi.org/10.12795/ revistafuentes.2016.18.2.07

Lyu, M. \& Gill, D. (2011). Perceived physical competence, enjoyment and effort in same-sex and coeducational physical education classes. Educational Psychology., 31 (2), 247-260. 
Martín Horcajo, M. (2006). Contribución del feminismo de la diferencia sexual a los análisis de género en el deporte. Revista Internacional de Sociologia. 44, 111-131.

Merino, I. (2013). Mide tu ritmo cardiaco desde iphone con Runtastic Heart Rate Pro. Recuperado de www.hipertextual.com

Moreno, J. A., Cervelló, E. y Martínez, A. (2007). Measuring self-determination motivation in a physical fitness setting: validation of the Behavioral Regulation in Exercise Questionnaire-2 in a Spanish sample. The Journal of Sport Medicine and Physical Fitness. 47 (3), 366-378.

Mouratidis, A.A., Vansteenkiste, M., Lente, W. \& Sideridis, G. (2011). Variation in vitality and interest-enjoyment as a function of class-to-class need-supportive teaching and pupil's autonomous motivation. Journal of Educational Psychology. 103 (2), 353-366.

Muñoz et al. (2012). Programa para el desarrollo de actitudes de igualdad de género en clases de educación física en escolares. Educación XX1,15 (2).

Pate et al. (2006). Promoting physical activity in children and youth: A leadership role for schools: A scientific statement from the American Heart Association Council on nutrition, physical activity, and metabolism (Physical Activity Committee) in collaboration with the councils on cardiovascular disease in the young and cardiovascular nursing. Circulation, 114 (11), 1214-1224.

Pérez Pueyo, Á. (2016). El Estilo Actitudinal en Educación Física: Evolución en los últimos 20 años. RETOS. Nuevas Tendencias en Educación Física, Deporte y Recreación, (29), 207-215.

Ruíz López, C.I. (2015). ¿Se han introducido las tic en la formación inicial de docentes? estudio de caso en el centro de magisterio "Virgen de Europa" . Revista Fuentes, 16,131-154 . D.O.I.: http://dx.doi.org/10.12795/revistafuentes.2015.116.06

Sevil, J., Abós, A., Julián, J.A., Murillo, B. \& García-González, L. (2015). Género y motivación situacional en Educación Física: claves para el desarrollo de estrategias de intervención. RICYDE. 11, 281-296.

Sicilia, A., Ferriz, R., Trigueros, R. \& González-Cutre, D. (2014). Spanish Adaptation and Validation of the Physical Activity Class Satisfaction Questionnaire (PACSQ). Universitas Psychologica, 13(4), 1321-1332.

Tahara, A.K. \& Darido, S.C. (2016) Tecnologias da Informação e Comunicação (TIC) e a educação física nas escolas. Corpoconsciência, 20(3),68-76.

Thyer, B.A. (2012). Quasi-experimental. Research Designs. New York: Oxford University Press.

Vansteenkiste, M. et al. (2012). Dentifying configurations of perceived teacher autonomy support and structure: Associations with self-regulated learning, motivation and problem behavior. Learning and Instruction, 22, 431-439

Vázquez, V. (2010). La perspectiva de la ética del cuidado: Una forma diferente de hacer educación. Educación XX1, 13 (1), 177-197

Volman M. (2005). Variety of roles for a new type of teacher. Educational technology and the teacher profession. Teacher and Teacher Education. 21 (1), 15-31.

\section{Para citar este artículo}

Sánchez Sánchez, L. \& Espada Mateos, M. (2018). Evaluación de un programa de intervención basado en el uso de las TIC para aumentar la motivación del alumnado en Educación Física. Revista Fuentes, 20(1), 77-86. [Fecha de consulta: dd/mm/aa]. doi: http://dx.doi.org/10.12795/revistafuentes.2017.v20.i1.05 